\title{
Interventions in Social Networks: Impact on Mood and Network Dynamics
}

\author{
DANICA VUKADINOVIĆ GREETHAM ${ }^{1}$, ABHIJIT SENGUPTA², ROBERT \\ HURLING $^{3}$, and JOY WILKINSON ${ }^{3}$ \\ ${ }^{1}$ Centre for the Mathematics of Human Behaviour, University of Reading \\ ${ }^{2}$ Essex Business School, University of Essex \\ ${ }^{3}$ Unilever R\&D Colworth, UK
}

\begin{abstract}
Results from two studies on longitudinal friendship networks are presented, exploring the impact of a gratitude intervention on positive and negative affect dynamics in a social network. The gratitude intervention had been previously shown to increase positive affect and decrease negative affect in an individual but dynamic group effects have not been considered. In the first study the intervention was administered to the whole network. In the second study two social networks are considered and in each only a subset of individuals, initially low/high in negative affect respectively received the intervention as 'agents of change'. Data was analyzed using stochastic actor based modelling techniques to identify resulting network changes, impact on positive and negative affect and potential contagion of mood within the group. The first study found a group level increase in positive and a decrease in negative affect. Homophily was detected with regard to positive and negative affect but no evidence of contagion was found. The network itself became more volatile along with a fall in rate of change of negative affect. Centrality measures indicated that the best broadcasters were the individuals with the least negative affect levels at the beginning of the study. In the second study, the positive and negative affect levels for the whole group depended on the initial levels of negative affect of the intervention recipients. There was evidence of positive affect contagion in the group where intervention recipients had low initial level of negative affect and contagion in negative affect for the group where recipients had initially high level of negative affect.
\end{abstract}


Keywords longitudinal networks; stochastic actor modelling; Katz centrality; positive and negative affect; network interventions.

\section{Introduction}

Collective mood of individuals in a social group and its relationship with the dynamics of the underlying network is a fascinating topic from a research point of view, but one which has mostly proved challenging in terms of a precise explanation of its mechanisms. Although there exists some prior knowledge on how emotion spreads in groups (e.g. group members mimicking the leader's emotions), it has been noted in a comprehensive review of this literature that "controlled research on emotion contagion within groups is actually quite sparse" (Niedenthal and Brauer, 2012). In fact, the authors go further to indicate that this sparsity may be attributed to the difficulty of designing studies which can provide sufficiently rich data sets for analysis.

Although not part of controlled experiments, recent availability of large data-sets of human to human communication obtained from online social networking platforms (such as Twitter, Facebook etc.), reveal evidence of correlation in levels of both positive and negative emotion between individuals and those they are directly connected to Quercia et al. (2012). Analysis of a large amount cross-cultural Twitter data (Golder and Macy, 2011) confirmed the existence of a daily and weekly rhythm of aggregated mood, and indicated correlation between friendship/follower relationships and positive/negative emotions of Twitter users across different cultures. A very recent study on Facebook data (Kramer et al., 2014) also found evidence of large scale emotional contagion among users in terms negativity and positivity of emotional content of their news feeds.

This emerging body of literature proves beyond doubt that mood and emotion are not only correlated across social networks, but that the very structure and nature of these networks might have an impact on the psychology of an individual, and as such, it may vary over time. Hence, it is natural that a complex systems approach can be applied when examining the dynamic nature of such phenomena and its interaction with underlying social networks. Large online social network data does provide us with a method of identifying and quantifying the dynamics of mood and emotion, but as indicated above (Niedenthal and Brauer, 2012), we 
have yet to understand the exact nature of how they interact with underlying social structures in a group or in a network. This paper is one such attempt, at identifying this relationship and examining it in the context of external events which act as "interventions" (see below).

Mood itself is a complex phenomenon which is difficult to measure in individuals (Fordyce, 2005; Gray and Watson, 2007). The social psychology literature often sees the use of Positive Affect (PA) and Negative Affect (NA) to connect mood and well-being (DeNeve and Cooper, 1998; Tellegen, 1985). These are constructs which are relatively easy to measure, have been established as independent of each other (Diener and Emmons, 1984) and follow independent short-term dynamics (Greetham et al., 2011). Gratitude, a psychological construct, has been suggested as being the general orientation towards appreciating the "positives" in life Wood et al. (2010). Gratitude interventions have been developed in order to encourage positive perspectives towards life in individuals, and have been shown to increase daily PA and decrease daily NA (Emmons and McCullough, 003a). Some limited amount of research has also been carried out involving the spread of PA and NA - for instance, through organizational networks (Totterdell et al., 2004). In one of the first of its kind in this line of research, this paper presents two independent studies, both of which aim to investigate how interventions within socially networked groups impact PA and NA, both directly as well as indirectly, through the resulting changes in the underlying network dynamics.

Psychological interventions are often carried out within groups of individuals to induce certain desired behaviors among its members (Norman et al., 2007; Michie, 2008; Abraham and Michie, 2008). In the analysis of the impact of these interventions however, key phenomena such as intra-group dynamics, impact of these interventions on the structure of the underlying social network and the feedback effects of the underlying structural changes on behaviour are almost always ignored. Very few studies have actually attempted to link the effect of gratitude interventions on individuals with the wider social context and relationship structure within which these individuals usually exist.

In fact, interventions within a social network can be a powerful tool to induce desired behaviors (Valente, 2012; Cross and Parker, 2004). This paper aims to fill this gap in the literature, by reporting on the results from two real life intervention studies which take into account the underlying network structure and dynamics, and examine how they in turn impact behavioural constructs (in this case levels of PA and NA) within the population. Additionally, 
we recognize that it may not be feasible to administer an intervention to all members of a group forming the social network - for instance, in case of policy interventions carried out on large groups. To that end, we also investigate the impact of administering the intervention to a small number of specific intervention nodes - who we refer to as agents of change within the network. The results are encouraging and in some instances surprising, and hence point towards a large number of possible avenues that can extend the research presented here. As we witness the development of this "emerging science of network based intervention" (Valente, 2012), we recognize that there is still lot of work to be done in measuring short and long term impact of these interventions within social networks and optimization of the same.

The main aims of this paper are summarized as follows:

- Investigate the results of a gratitude based intervention on an evolving social network and the relationship between a network's structure and dynamics on mood related constructs, viz. Positive Affect and Negative Affect.

- Explore the impact of choosing target individuals (agents of change) for selective intervention within a network and test the choice of low negative affect as a predictor for communicability of individuals.

- Attempt to separate mood influence (contagion) effects from selection (homophily) effects on the evolving network as a result of the intervention.

In Section 2, we provide a brief background on the underlying theory on both social network analysis as well as on the PA and NA constructs used as the basis of evaluating the effects of intervention. In Section 3, we explain our methodology and estimation procedures used in analyzing the results from the studies. In Section 4, we provide the description and results of Study I, which examines the impact of providing an intervention to the whole population. Subsequently, in Section 5, the results are provided from Study II, which restricts the intervention to a small minority of individuals in the network, with the aim of detecting contagion, if any. Finally, we conclude with a discussion of our results along with some directions for the future work ${ }^{1}$.

\footnotetext{
${ }^{1}$ All data collected as part of the studies are owned by Unilever and hence cannot be released on request. However, the authors are happy to provide the "input matrices" derived from the raw data and used in SIENA, upon request.
} 


\section{Previous work}

\subsection{Network interventions}

A network intervention uses social network data to accelerate behavior change or make it more sustainable across the targeted population. In a very recent review (Valente, 2012), four main strategies of network interventions are identified: individual-picking agents of change based on their position in network (central, peripheral, ability to bridge two communities, etc.) or their behavioral characteristics (e.g. extrovert, smoker/non-smoker etc.); segmentation simultaneous behavior change for subgroups, thus requiring community detection, but allowing application of different interventions on the different parts of network (e.g. intervening in core versus periphery); induction - stimulating or enforcing new ties in order to propagate a message or behavior through the network; alteration - creating or deleting network nodes and/or ties and/or re-wiring existing ties.

Depending on context, availability and character of network data, the nature of the network itself and the intervention being considered, each of these strategies offer a wide choice of algorithms and processes. In this work we use the first strategy, picking individuals on the network as the agents of change while assuming knowledge about the whole network on part of the mechanism designer. The aim is then to optimize the choice of individuals, or more precisely to measure the influence of choice on the intervention results.

Previous work (Valente and Pumpuang, 2007) indicates that identification and recruitment of opinion leaders/key players who can act as champions for the desired intervention in order to accelerate behavior, is key. And for this purpose, one has to compute the appropriate centrality measures (local or global) across the network. It is this measure of centrality, which quantifies the relative importance of a node and determines its involvement within a network. The literature in social network analysis has proposed a number of different centrality measures, and have tested and compared them on undirected, directed and weighted networks. See Borgatti and Everett (2006), Opsahl et al. (2010) for reviews. However, it is only recently that research has focused on centrality in dynamic, evolving networks (Grindrod et al., 2011).

For static networks, Katz centrality (Katz, 1953) computes the relative influence of a node within a network by measuring the number of the immediate neighbors, and all the other nodes in the network that connect to the node under consideration through the immediate neighbors. 
Walks made to distant neighbors are penalized by an attenuation factor. This concept was recently revisited in Estrada and Hatano (2008), Grindrod et al. (2011). Centrality across time-steps is based on the extension of Katz centrality to evolving networks. For example, if $\mathrm{A}$ and $\mathrm{B}$ interact on Monday and B and C interact on Tuesday then information can be passed from A to $\mathrm{C}$ but not vice versa, which is normally overlooked by looking at the aggregated networks and ignoring time dimension. This asymmetry gives rise to two types of centrality indices during a time-window - the first quantifies the ability of an individual to pass a message onwards, and is called broadcast index, and the second, quantifies the ability to listen or receive message and is called receiver index.

\subsection{Positive and Negative Affect}

As mentioned earlier, Positive Affect (PA) and Negative Affect (NA) are connected to mood and well-being in the social psychology literature (DeNeve and Cooper, 1998; Tellegen, 1985). In a study involving emotional contagion of small working groups of 2 to 4 members (Barsade, 2002), the author reports "a robust finding of group contagion", and also that there was no difference in the degree of contagion of negative and positive moods/emotions. The emotion contagion was also shown to influence subsequent group dynamics. In two studies investigating the relationship between organizational networks and employees' affect (Totterdell et al., 2004), PA and NA were shown to spread within work groups. Similarity of affect between employees depended on the presence of work ties and structural equivalence. Affect was also related to the size and density of employees' work networks. Furthermore, they investigated a merger of two organizational groups, and found that negative changes in employees' affect were related to having fewer cross-divisional ties and to experiencing greater reductions in network density. On a similar note, a recent study analyzing large amount of Twitter and Facebook data Quercia et al. (2012) shows correlation between friendship/follower relations and positive/negative mood of Twitter users.

In an interesting piece of work examining the daily and seasonal dynamics of PA and NA, obtained from a large cross-cultural set of Twitter data (Golder and Macy, 2011), the authors identified individual-level diurnal and seasonal mood rhythms that are common across different cultures. In accordance with previous small-scale lab studies, they found that individuals are most positive in the morning but mood deteriorates as the day progresses. People are overall 
happier on weekends, and diurnal rhythms over weekdays are nearly identical for both PA and NA. While there is not much difference within the weekdays, PA is higher significantly on weekends. This difference between weekend and weekdays is confirmed in countries where weekend is on Fridays and Saturdays as well. Seasonal change in baseline positive affect varies with change in day-length which supports previous findings that 'winter blues' is related to diminished PA but not increased NA.

\section{$2.3 \quad$ Longitudinal network analysis}

There exists a lot of on-going research and debate on correlation versus causation in every discipline involving empirical research. This is especially relevant in the case of research involving data from social networks. It is often relatively easy to identify correlations between constructs involving network dynamics, but it is more difficult to tease out the direction of causality within these constructs and their interplay with the underlying network structure. This is compounded if the structure is dynamic, i.e. evolving through the time.

Fortunately, the recent development of empirical methods based on stochastic actor-based models for network dynamics implemented in SIENA - Simulation Investigation for Empirical Network Analysis (Snijders et al., 2010; Snijders, 2005) have enabled study of co-evolving network dynamics and behaviour of its members, allowing us to differentiate between two types of processes: social selection and social influence. Selection processes describe how actors choose other actors and form ties, based on their assigned attributes. Influence processes describe dynamics of actors' observed qualities or attributes (PA and NA, in our case) and its influence on other members of the network, connected either directly or indirectly. In any longitudinal network based study, it is critical that both of these are accounted for, as they represent distinctly different processes and have different impact on behaviour.

SIENA is fast becoming a standard tool of choice when examining longitudinal network data and have been used in a number of studies. In a recent pilot study involving small groups and longitudinal networks (Greetham et al., 2011), SIENA was used to analyse dynamics of mood over networks, changes in PA and NA, and the inter-relationships between the two. One of the main results was significant difference in the short-term dynamics of positive and negative affect. In fact, this forms the basis of the design of our studies reported in this paper. Other notable uses of SIENA can be found in Huisman (2013), Schaefer et al. (2013) and 
Ellwardt et al. (2012).

\section{Methodology}

We explain in more detail network modelling and analysis techniques that were used to analyze the results of the studies.

\subsection{SIENA}

As mentioned above, we used SIENA Snijders et al. (2010), Ripley and Snijders (2011), a stochastic actor-based framework to model the simultaneous evolution of network structure and behavior/individual characteristics of nodes in the network. SIENA is able to incorporate actor covariates, dyadic covariates as well as characteristics of the underlying network to statistically model the process of network evolution and behavior at the same time. As its input, SIENA requires "network ties" data from a number of observation moments, where each moment is labelled as a 'panel wave'. The minimum number of such waves required is 2 and is generally kept below 10. A 'tie' in SIENA is represented as a binary variable $x_{i j}$ which takes value 1 if there exists a link initiated by $i$ (the ego) to $j$ (the alter) and 0 otherwise. The term 'initiation' is key and hence the reason behind calling these models 'actor based'. The term stochastic arises from the fact that network ties are considered as 'states' and hence the dynamics of the network is interpreted as a Markov process. The tie variables, represented by a $n \times n$ adjacency matrix for a given time period, represents a panel wave and is the key input into the model.

For model identification, we use forward and backward stepwise procedures (as recommended in Snijders et al. (2010) and Ripley and Snijders (2011)), to test the impact of including/excluding specific independent variables on the overall goodness of fit and statistical significance. Since the stochastic model itself is too complex for classical estimation methods, SIENA uses simulation based procedure to model the network change process. The references mentioned above contain all the details of the estimation procedure, its strengths and weaknesses in great detail and would be useful for the interested reader. We provide additional details on the estimation procedure, a list of variables considered for inclusion and other details in the Appendix of this paper. Additionally, Schaefer et al. (2010) provides an interesting 
application of SIENA in exploring network formation and dynamics among groups of children.

\subsection{Centrality in evolving networks - communicability}

We used the concept of communicability in evolving networks (Grindrod et al., 2011) to identify the key members within a network. For evolving (longitudinal) networks, such as the ones studied here, we assume that the set of nodes is fixed, i.e. no new nodes are added or old ones destroyed, and that edges can evolve (i.e. they can be created, deleted or maintain status quo). At any time step $t$, let $A_{t}$ be the adjacency matrix of the network. Based on $M$ adjacency matrices, we can compute a communicability matrix

$$
Q=\prod_{t=0}^{M}\left(I-\alpha A_{t}\right)^{-1},
$$

where $I$ is identity matrix, $\alpha=\frac{1}{2 \max \left(\rho\left(A_{t}\right)\right)}$ and $t=0, \ldots, M$ are consecutive time-steps and $\rho\left(A_{t}\right)$ is the largest eigenvalue of $A_{t}^{2}$.

$Q$ is a square matrix, with rows and columns representing nodes or individuals in the network. Intuitively, it represents the number of walks of all possible lengths between every pair of vertices, where longer walks are penalized more. Once computed for a set of given time-step matrices, the element $Q_{i, j}$ summarizes how well information passes between the two actors $i$ and $j$, given the evolving nature of the network, represented through adjacency matrices $A_{t}$. The $k^{\text {th }}$ row and column sums, each represents a measure of communicability for actor $k$. The row sum represents the broadcast index while the column sum measures the receive index. As the respective names suggest, they measure how well actor $k$ is able to broadcast and receive messages over the network.

\section{Study I: Uniform Intervention}

This study examined the impact of an intervention within a dynamic social network, where all actors in the social network received an intervention aimed at improving their wellbeing. The aim was to identify the key attributes of a network and the individuals within the network, which influenced how the intervention impacted the overall behavioural traits (PA and NA) of all individuals. It also aimed to identify the impact of the intervention on the network structure

\footnotetext{
${ }^{2}$ Note that $\alpha<\frac{1}{\rho\left(A_{t}\right)} \forall t=0, \ldots, M$, for the inverse to exist.
} 
itself, in terms of the number and nature of ties at various nodes of the network. Note that, given the nature of the study, there was no explicit control group to benchmark the results against, but the pre-intervention measures of behavioural traits were used as a baseline against the post-intervention measures. We intended to provide an initial understanding of network dynamics in the presence of interventions, which would guide a more detailed exploration.

\subsection{Study Design}

This study consisted of 89 participants, who were all second year students from a UK University located in the midlands. The study itself ran for 4 consecutive days (Days 1 to 4, Monday to Thursday) with pre (baseline) and post study measures taken on separate days (Day 0 and Day 5 respectively, Wednesdays before and after the week in which the intervention took place ). The intervention on the afternoon of Day 3 and 4 consisted of writing up three things that one is grateful for that day, while eating an ice-cream. Participants were given an ice-cream voucher which could be exchanged for an ice-cream in the campus cafeteria, where a drop-box for filled out questionnaires was also located.

A number of background, behavioural and network related measures were taken before, during and after the study. First of all, the gender, age and ethnicity of participants were recorded. During the 4 main days of the study, the participants were also asked to record their respective PA and NA using a 10 item Positive Affect and Negative Affect Scale (PANAS) (see D) twice, once in the afternoon and once in the evening. On days 3 and 4, this was done in conjunction with the gratitude intervention, i.e. the participants were asked to complete a "Three Good Things" exercise - write down three things they are grateful for that day while eating the ice cream during the afternoon ${ }^{3}$. Additionally, the baseline and post study measures, taken on days 0 and 5, consisted of the following: trait Gratitude using a 6 item Gratitude questionnaire Emmons and McCullough (003b), subjective Happiness using the 4 item Subjective Happiness Scale, Satisfaction with Life Scale (SWLS) using the 5 item scale (Diener et al., 1985), Relatedness using Need Satisfaction in Relationships 9 item scale(La Guardia et al., 2000), Loneliness using 10 item UCLA Loneliness Scale (Russell and Cutrona, 1980) and PA, NA using a 20 item PANAS scale (see D).

\footnotetext{
${ }^{3}$ While the PA and NA measures were taken twice during the day, we used the evening measure only for the study.
} 
Secondly, all participants were asked to record their daily interactions (frequency and duration) with others within the network, which would provide the data to estimate ties among the participants. A maximum limit of 10 interactions was set for each part of the day (morning, afternoon, evening) to constrain the task for participants and to ease data analysis (and this limit was reached on five occasions during the main 4 days of the study). Each recorded interaction detailed the name and description (friend or acquaintance) of the contact, the time and duration of interaction, the type of interaction (face to face, phone, SMS, email or online) and finally, a rating on the interaction from very negative to very positive (5 point scale). We constructed an evolving network in 4 time-step (4 days) from the daily interaction diaries, denoting an existence of a link from $\mathrm{A}$ to $\mathrm{B}$ at time $t$, if there was an entry in A's diary denoting a contact with B on a day $t$. We also constructed 4 weighted daily matrices where an entry $w_{A B}$ denoted total number of minutes recorded by $\mathrm{A}$ that was spent in contacts with B during day t. Due to the extensive nature of the study, students were compensated at the end of the study. The study was approved by the relevant University Ethics Committee.

\subsection{Aims and Hypotheses}

The data generated from Study 1 was used to examine two types of phenomena. The first phenomenon relates to the effect of the intervention on the network dynamics, particularly how it impacts communication between individuals within the network and whether there is evidence of spillover effects, across individuals, relating to overall behavioural traits PA and $\mathrm{NA}$ - such as homophily ${ }^{4}$ and contagion. The second phenomenon relates to the actual impact on PA and NA within individuals - i.e. whether this intervention, which aimed at improving well-being was actually successful, measured through overall levels of PA and NA. These can be summarized in the following 3 hypotheses, which we test using the data from the study.

H1 Affect homophily is present within the social network.

H2 The intervention is associated with contagion of PA and NA across the group, through the underlying evolving network.

\footnotetext{
${ }^{4}$ Homophily is the tendency of individuals to make links and associate with others most similar to ones own self (see McPherson et al. (2001)).
} 
H3 The intervention is associated with increase in group level PA and decrease in group level NA overall.

\subsection{Results}

All network based analysis was done using the $\mathrm{R}$ based SIENA package, which uses actor based models to analyse longitudinal evolving networks. The analysis took into account two separate, but co-evolving processes. Social interaction, which represents the impact of endogenous structural and behavioral variables, as well as exogenous covariate variables, on the likelihood of tie formation from the ego towards the alter. Social influence, on the other hand, examines the evolution of the endogenous behavioral variables as a function of network characteristics and other exogenous variables. SIENA examines the co-evolution of these two mutually dependant phenomena. More details about the estimation algorithms and procedures can be found in Snijders et al. (2010), Ripley and Snijders (2011). We used 2000 iterations for the estimation of the covariance matrix and all t-ratios were less than 0.1 indicating excellent convergence properties between observed and expected values (obtained by simulations' averages) of important features. Jaccard coefficients ${ }^{5}$ between the 4 consecutive days were $0.417,0.351$ and 0.322 respectively - on the lower side, but still within acceptable limits of network changes over the duration of the study. SIENA is used to test $\mathrm{H} 1$ and $\mathrm{H} 2$, while a more straightforward statistical comparison between baseline and post intervention measures is used when testing H3. For the benefit of a reader unfamiliar with the terminology, we provide a brief description of all variables in $\mathrm{A}$.

\subsubsection{Dynamics of network and affects}

The following structural variables were used as controls in the analysis: network rate, a basic characteristic of network evolution which models the speed by which each actor gets an opportunity to change ties; reciprocity, which measures the tendency to reciprocate ties (in

\footnotetext{
${ }^{5}$ The Jaccard coefficients are used to measure the similarity between the data collection points, or waves. The waves should ideally not be too different from each other, as that might imply structural changes not brought about through an endogenous process. It is measured between two waves by, $\frac{N_{11}}{N_{11}+N_{01}+N_{10}}$, where $N_{11}$ is the number of ties present in both waves, $N_{01}$ is the number of ties newly created and $N_{10}$ is the number of ties terminated. Ideally, the coefficient should be above 0.3. See Snijders et al. (2010) for more details.
} 
our case a recall of communication); outdegree, which measures the basic tendency to have ties at all; and transitive triplets, which measures the tendency toward network closure within groups of three. Additionally, we also included PA similarity, NA similarity, PA ego, PA alter, NA ego, NA alter. The covariate similarity effect measures tendency of an actor to prefer ties to others with similar values on this variable (thus measuring homophily, i.e. contributing to similarity not by changing the variable but by changing the network); the covariate-ego/alter effect measure the tendency that an actor with a higher value on those variables increases her out-degree/in-degree resp. more rapidly. Among exogenous covariate effects, we included gender, age, ethnicity, Satisfaction with Life (SWLS), Happiness, trait Gratitude, Relatedness and Loneliness on network as well.

Positive and negative affect were self-reported each day in the afternoon and the evening (see D). They were measured using the 10 item scale, but were recoded to scale 1-4 for use in SIENA by transforming original range 5-25 to 1-4 in the following way: all values between 5 to 10 were assigned 1, 11 to 15 were assigned 2, 16 to 20 were assigned 3 and 21 to 25 were assigned 4. For the PA scores, there was a significant change of up to 2 points on the 5-25 scale on average between afternoon and evening scores on each day, with people recording higher scores in afternoons than in evenings. The change in NA scores from afternoons to evenings was not as strong (less than 1 point in average), but still significant across all days, showing a small drop from afternoon to evening. The data indicates that affect scores generally decreased from afternoon to evening, with a greater drop in positive than negative affect. However, these changes were small enough to ensure that most of the values stayed within the same category after the recoding was done. We found no evidence that the change in positive and negative affect between baseline and intervention days was different for the afternoon versus the evening measures. Hence we used just the evening measures for the rest of the analysis.

For the purpose of evaluating the key parameters affecting social influence, we included rate, linear shape, quadratic shape, average alter and the exogenous effects mentioned above, on PA and NA. The linear shape effect measures the basic trend toward high values, and the quadratic shape measures the effect of the behavior on itself, i.e. presence of a feedback effect - to assess if behaviour is self-reinforcing or self-correcting. The average alter effect measures the tendency of contagion, that is, the tendency of actors to become more like their neighbours, by actually changing behaviour rather than changing ties. 
Following the procedure set out in Snijders et al. (2010), an iterative method was used to decide on inclusion or exclusion of variables within the estimated models. Table 1 presents the results from the final iteration: parameter estimates and standard errors for all variables with potential impact on both the network dynamics (selection) and behaviour (influence) related measures ${ }^{6}$. We used goodness of fit tests implemented in RSiena to check how well the simulated networks in our model match the observed networks with respect to the distribution of indegree, outdegree and PA and NA values ${ }^{7}$. Both indegree and outdegree distributions were well fitted producing $p=0.773$ with Mahalonobis distance(MHD) of 56.975 and $p=0.762$, $\mathrm{MHD}=64.468$ respectively. The PA distribution was marginally good fit with $p=0.052$, $\mathrm{MHD}=16.477$ and NA a very good fit with $p=0.789$ and $\mathrm{MHD}=6.935$.

In terms of overall network dynamics, all three network effects - reciprocity, outdegree and transitivity - were revealed to be significant in the data. With respect to the baseline measures, only SWLS alter had a significant influence on the network structure, with a positive coefficient for SWLS-alter. This indicates that participants with higher values of SWLS have being more likely to be approached by others (independently of past contacts). In terms of the behavioral variables, significant positive coefficients were obtained for PA and NA similarity as well, which imply that people preferred ties with others who have similar values of PA and NA independently (homophily).

To examine social influence processes, we tested for linear and quadratic shapes, total and average similarity ${ }^{8}$, indegree and outdegree, and average alter effect for both PA and NA. Results reveal significant positive coefficients on the quadratic term and non-positive coefficient on the linear term for both PA and NA. This implies a general tendency towards extreme values - i.e. high values of PA/NA tend to be pushed up and lower values are pulled down further over time. This result is similar to the one found in a previous study Greetham

\footnotetext{
${ }^{6}$ Here and in all subsequent Tables containing results of statistical analysis, statistical significance of 0.05 is indicated by two stars $\left({ }^{* *}\right)$ and of 0.01 is indicated by three stars $\left({ }^{* * *}\right)$ alongside the corresponding variable or estimate

${ }^{7}$ The sienaGOF() function implements Monte Carlo test based on the Mahalonobis distance to calculate relevant p-values. See Snijders et al. (2010) and the RSiena manual for more details.

${ }^{8}$ The average similarity effect measures the preference of actors to being similar regarding covariate to their alters, where the total influence of the alters is the same regardless of the number of alters. For the total similarity effect, the total influence of the alters is proportional to the number of alters.
} 
Table 1: Estimated effects and standard errors from final iteration of SIENA.

\begin{tabular}{ccc}
\hline Social Selection Effects & Estimate & S.E. \\
\hline network rate period 1 & 3.7854 & 0.5326 \\
network rate period 2 & 6.7361 & 1.1085 \\
network rate period 3 & 6.0325 & 0.9878 \\
outdegree** & -3.2496 & 0.1418 \\
reciprocity** & 2.8383 & 0.1625 \\
transitive triplets** & 0.5293 & 0.0548 \\
SWLS alter** & 0.1498 & 0.0717 \\
PA similarity** & 1.0274 & 0.5098 \\
NA similarity** & 1.7299 & 0.8313 \\
& & \\
\hline Social Influence Effects & Estimate & S.E. \\
\hline PA rate period 1 & 3.0714 & 0.7974 \\
PA rate period 2 & 2.4594 & 0.6358 \\
PA rate period 3 & 3.5815 & 1.3245 \\
PA linear shape & -0.3576 & 0.0871 \\
PA quadratic shape** & 0.0558 & 0.0683 \\
Relatedness on PA** & 0.1908 & 0.0795 \\
NA rate period 1 & 2.527 & 0.9604 \\
NA rate period 2 & 2.0307 & 0.9311 \\
NA rate period 3 & 1.4484 & 0.5072 \\
\hline NA linear shape** & -2.1642 & 0.2589 \\
quadratic shape** & 0.4405 & 0.1506 \\
Nade on NA** & -0.4797 & 0.2291 \\
\hline
\end{tabular}

et al. (2011). Moreover, no evidence of spillover or contagion in either PA or NA were found in significant proportions, in terms of average similarity, alter, in-degree or out-degree effects. Thus, we have two following results.

R I.1 PA and NA tend to be pushed towards extreme values. 
R I.2 The underlying social network exhibits homophily with respect to PA and NA, but no contagion could be observed.

Note that, given $\mathbf{R} \mathbf{I} \mathbf{2}$ we are able to establish $\mathbf{H 1}$ but not $\mathbf{H 2}$.

\subsubsection{Intervention versus Baseline}

We carried out a standard statistical comparison of means between pre and post intervention levels of PA and NA within the population. This is done in order to see if we can detect changes as a result of the intervention by measuring overall levels of PA and NA, ignoring the underlying network dynamics. We carry out a pair-wise comparison of each measure across Days 1 to 4 . Additionally, we compare Days 0 and 5 as well, to get an idea of the underlying trend. For making the comparisons, we use the non-parametric Wilcoxon Signed Rank test, which does not necessitate the strict assumptions of an underlying normal distribution and is appropriate for paired data. This approach does have the weakness that observations in our data may not be independent, given that the measures are taken across an evolving social network. Hence, the p-values may not be entirely correct as independence of observation pairs is a requirement of the Wilcoxon Signed Rank test. The p-values should be used for guidance only, and not be considered as a rigorous test of statistical significance ${ }^{9}$.

The results of the analysis done on the collected data is summarized in Table 2 in the form of p-values derived from each pair-wise comparison, where the null represents no change in the variable concerned, and the title of columns two and three represent the alternate hypotheses.

The p-values reported in Table 2 indicate that there is a statistically significant increase in Positive Affect post intervention, especially when the first and the last days of the study are compared (day 1 to 4 ). There is no evidence of increase in PA between days 1 and 2 or between 2 and 3. However, there is clear evidence of an increase in PA between days 3 and 4 - which can be linked to the repeated intervention on the last two days. Hence we can claim support for H3, although with the caveat on the independence of observations stated above. This is borne out by the pre and post study measures of PA as well (last row in Table 3).

However, the case of Negative Affect is more complicated. Whereas there is a statistically significant drop in NA between days 1 and 4, supporting the hypothesis, it is difficult to

\footnotetext{
${ }^{9}$ We thank and acknowledge one of the anonymous referees for pointing out this issue of independence of observations.
} 
Table 2: Wilcoxon Rank Sum Test p-values for pairwise comparison between days for PA and NA.

\begin{tabular}{ccc}
\hline Change from & PA increase & NA decrease \\
\hline Day 1 to 2 & 0.1026 & 0.3734 \\
Day 1 to 3 & 0.0978 & 0.0418 \\
Day 1 to 4 & $0.0008^{* * *}$ & $0.0034^{* * *}$ \\
Day 2 to 3 & 0.4689 & 0.0720 \\
Day 2 to 4 & 0.0628 & $0.0305^{* *}$ \\
Day 3 to 4 & $0.0087^{* * *}$ & 0.2857 \\
Day 0 to 5 & $<0.0001^{* * *}$ & 0.9948 \\
\hline
\end{tabular}

attribute this to the effect of the intervention as the evidence from the intermediate days is weak. However, there is a significant increase in NA between the pre and post study measures (with a p-value of 0.0001 when tested), which may be attributed to external confounding factors. Hence, the fact that there is some evidence of decrease in NA during the intermediate days is important, which leaves us with the following result, in support of H3:

R I.3 The intervention on days 3 and 4 coincides with an increase in PA and decrease in NA overall.

\subsection{Communicability and NA}

Communicability matrices were created from the 4 daily interaction adjacency matrices, where interactions were marked as 0 and 1 , and also where interactions were measured for their durations in minutes. We computed the broadcast and receive indices for both binary $(Q)$ and weighted matrices $\left(Q_{d u r}\right)$, where the weights were equal to the duration of communication in minutes. As mentioned above, the row and column sums of each of these matrices provides the broadcast and receiver indices respectively (both binary and weighted, depending on the matrix used).

The broadcast and receive indices were strongly correlated (coefficient of correlation $\sim$ 0.87). This can be explained by the lack of hierarchy in the group (i.e. there were no predesignated"receivers" and "broadcasters"). However, there was no significant correlation with 
the degree (which is defined as a total number of intercommunication instances for an actor), betweenness (all minimum paths between any two actors that pass through a particular actor), normalized degree or normalized betweenness of the friendship and acquaintanceship (friendship and acquaintanceship matrices obtained before and after study). Additionally, no significant correlation could be established between these indices and average PA and NA of the individuals (where the averages are taken for each person over the four days of the study).

However, one interesting pattern was revealed from the data, when the weighted broadcast and receiver indices were calculated. First of all, majority of actors had both weighted indices - broadcast-dur and receive-dur - equal to zero. However, those particular individuals with the top 5 broadcast-dur indices were also the ones with the minimum average NA (see Fig 1). This implied that, at least as far as this data was concerned, when the duration of communication is taken into account, the best "broadcasters" were also the people with the smallest possible levels of negative affect (note that the majority of the reported communication - i.e. on the average $70-80 \%$ each day - were face-to-face, approximately $5 \%$ was by phone, and the rest was digital). This finding, although surprising and difficult to explain, is still indicative enough to use in the next study, where we choose a selected few to whom we administer the intervention.

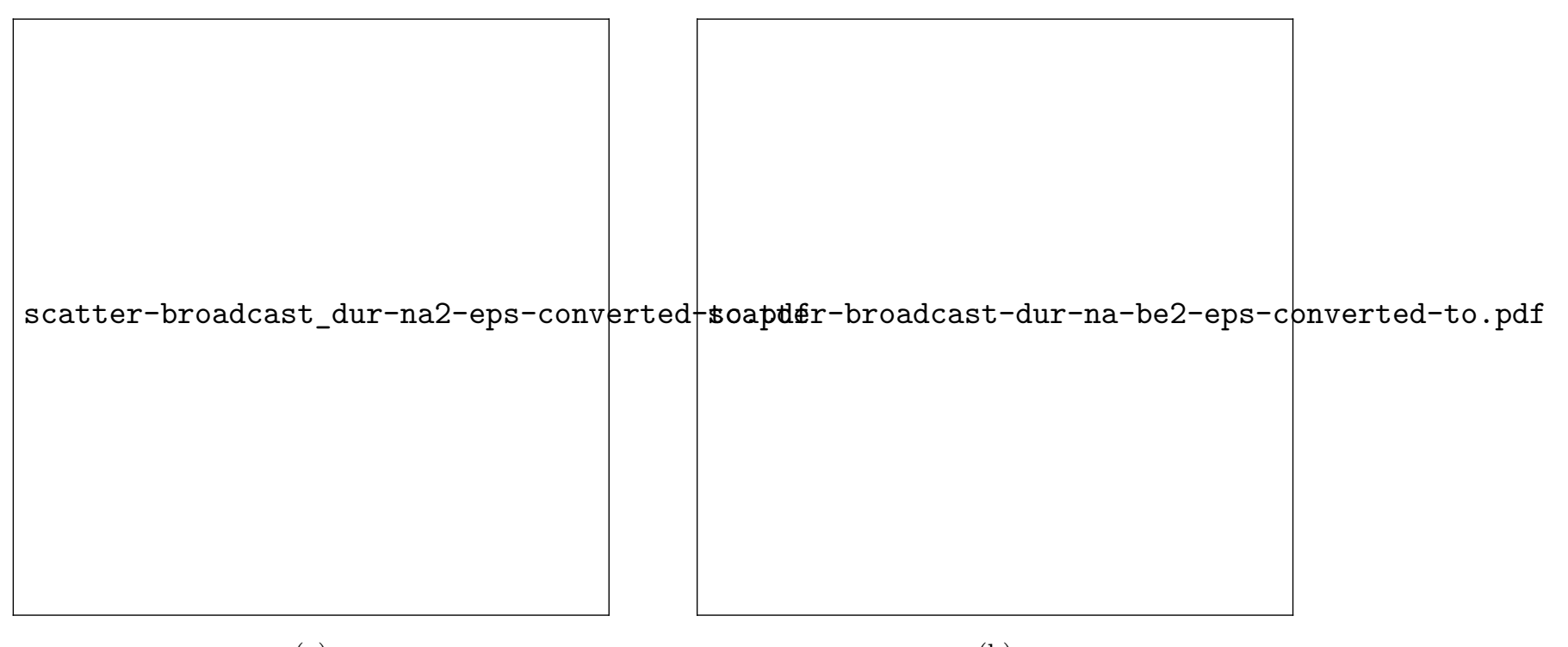

(a)

(b)

Figure 1: Panel (a): Scatter plot of duration weighted broadcast index versus 4-day average of NA . Panel (b): Scatter plot of duration weighted broadcast index versus NA before the study. 


\section{$5 \quad$ Study II: Selective Intervention}

This study extends Study I by investigating the effect on network dynamics and resulting implications on well being, when an intervention is administered to only a subset of individuals in a group, rather than targeting it to everyone. Administering an intervention to everyone in a population may be optimum in terms of ensuring the desired outcome (as seen in Study 1) but is usually not feasible for a number of reasons - costs, unavailability and lack of resources being the most obvious ones. This study examines the efficacy of using limited interventions within a network, with the aim of spreading the effect (or behaviour) to the whole population through contagion alone. To the best of our knowledge, this is one of the first studies of its kind, where limited psychological interventions are used to encourage contagion, and provides important directions for future network intervention studies of the same nature. The recent Facebook study reported in Kramer et al. (2014) bears some resemblance to ours, in that there is targeted intervention among test subjects, but it does not explore the causal mechanisms of the underlying dynamics of mood change (including feedback between the network change and behaviour) as well as impact of the initial mood state of the individuals receiving an intervention. Both these aspects are explored in this study.

In Study I, the gratitude and ice-cream interventions proved successful in terms of raising overall emotional well-being (by raising group level PA and reducing group level NA), when the intervention was applied to the whole group. We also saw evidence of homophily in the contact network where individuals chose others with similar levels of both positive and negative affect to communicate to, but did not find any significant evidence for contagion (transfer of positive/negative affect within a group). In this study a limited number of individuals were targeted with the intervention in order to measure significant spillover effects, if there are any. Our finding in Study I, that the best broadcasters are always low NA individuals, is used in this study to identify the so called "agents of change" - individuals who receive the intervention.

\section{$5.1 \quad$ Study Design}

We recruited two groups of second year students enrolled in a degree course from a university in the south east of the United Kingdom. Students in each of these groups, which are labelled 
as A and B, were chosen so that they participate in similar activities/courses inside the group, but the two groups do not overlap. Participants were asked for their consent and were paid for their participation, given the intense requirements of the study. Ethical approval was obtained from the University's ethics committee. In group A, 62 participants completed the study and in group B, 65 participants. All baseline, pre, post-study and daily measures were the same as in the Study I, with two notable exceptions. Firstly, 10-item PANAS was measured just in the evening (the more crucial measure which took into account all daily interactions). Given the finding in Study 1 regarding the drop in affect measures in the evenings compared to afternoons, a single evening measure not only takes into account all interactions, but eliminates diurnal bias as well. Additionally, the reporting burden for participants was lower as well. Secondly, as mentioned above, not all the participants received an intervention. The same intervention was administered on the third and fourth days of the study, but only to approximately a quarter of the participants in each group.

The main study ran for 4 days (Monday to Thursday), while pre and post measurements were taken on the Wednesday the week before, and a post study measures were taken on the Wednesday the week after. In group A, 15 individuals were chosen for the intervention (approx. 24\%) and these were specifically individuals with the smallest levels of negative affect on the first day of the study. In the group B, 13 individuals were chosen for the intervention (approx. 20\%) and these were individuals with the highest levels of negative affect on the first day of the study. No other network-specific criteria (such as degree, betweenness etc.) was used in the selection of these agents of change.

\subsection{Aims and Hypotheses}

It was hypothesised in Barsade (2002) that "when people are feeling low energy, and unpleasant, they become more internally oriented, withdrawn from the group, with less opportunity to influence other group members". Given that the agents of change are chosen on different criteria in the two groups, we expected to find differences between the two in terms of contagion effects, which forms the basis of the following hypothesis.

H4 The existence, nature and extent of contagion of PA and NA in the whole group depends on the original mood state of the individuals receiving the intervention. 
If true, the above would prove critical in designing interventions in large groups. Unlike the interventions investigated in areas such as disease control (see Duerr et al. (2007)), where the structure of the network is critical, the above would imply that the emotional states of these agents at the time of the intervention would also have to be taken into account in order to maximize the chances of success.

\subsection{Results}

\subsubsection{Dynamics of networks and affects}

Once again, we tested for both types of phenomena - selection processes, which have to do with the evolving structure of a social network and influence process, that relate to the measured behaviours (PA and NA). We used goodness of fit function in RSiena to check how well the simulated networks in our model match the observed networks regarding indegree, outdegree and PA and NA values distribution. For group A, indegree and outdegree distributions were well fitted producing $p=0.929$ with Mahalonobis distance(MHD) of 17.996 and $p=0.495$, $\mathrm{MHD}=17.945$ respectively. The PA distribution was marginal fit with $p=0.015, \mathrm{MHD}=19$ and NA a very good fit with $p=0.761$ and $\mathrm{MHD}=3.621$. For group $\mathrm{B}$, indegree and outdegree distributions were relatively well fitted producing $p=0.187$ with Mahalonobis distance(MHD) of 33.346 and $p=0.276, \mathrm{MHD}=28.358$ respectively. The $\mathrm{PA}$ and NA distribution were excellent fits with $p=0.961, \mathrm{MHD}=3.743$ for $\mathrm{PA}$ and $p=1$ and $\mathrm{MHD}=2.564$ for $\mathrm{NA}$. We used SIENA time test to test time heterogeneity, and included time dummies for outdegree and transitive triplets for time periods 2 and 3 for group A, and outdegree, transitive triplets and PA alter for time period 2 for group B.

R II.1 In Group A, higher values of PA implied more outgoing ties but less incoming ties. No such effect was found in Group B.

This can be seen in results Tables 3 and 4. For Group A (Table 3), PA alter is significant (at 0.05 level) and has a negative coefficient. Whereas, for Group B (Table 4), its PA alter has a negative coefficient as well but is not significant. This implies that in Group A, the individuals with higher PA tended to be contacted by less people on the average, but no such result could be established for Group B. In fact, in Group B what mattered for incoming ties was whether 
one received the intervention or not (as seen in the positive coefficient of intervention alter in Table 4).

Note that no significant evidence of homophily was found, which can be seen in the absence of significant PA similarity and NA similarity effects for both groups (in fact, leading to convergence problems when the NA similarity covariate was included for group B). We address the implications of these findings in more detail in the Discussion section.

Table 3: Group A Selection Effects: Estimates and standard errors

\begin{tabular}{ccc}
\hline Effect & Estimate & s.e. \\
\hline network rate period $1^{* *}$ & 4.2673 & 0.6482 \\
network rate period $2^{* *}$ & 4.5105 & 0.7070 \\
network rate period $3^{* *}$ & 4.8820 & 0.7627 \\
outdegree** & -2.9150 & 0.1267 \\
reciprocity** & 2.4119 & 0.2211 \\
transitive triplets** & 0.4901 & 0.0559 \\
int alter & 0.2270 & 0.1538 \\
PA alter** & -0.3988 & 0.1860 \\
time dummy 2 & -0.2370 & 0.3009 \\
time dummy 3 & 0.1778 & 0.2504 \\
time dummy 2 x reciprocity & 0.1955 & 0.5754 \\
time dummy 3 x reciprocity & 0.1083 & 0.5407 \\
time dummy $2 \times$ transitivity triplets** & -0.3171 & 0.1371 \\
time dummy $3 \times$ transitivity triplets & 0.0134 & 0.1505 \\
\hline
\end{tabular}

We investigated PA ego, alter and similarity effects in Tables 5 and 6 in order to understand how the effects work. See Ripley and Snijders (2011) for the details on creating ego-alter selection tables and the formulae containing ego, alter and similarity effects that is used. The tables gives the preference for similar alters. We see in Table 5 that in all rows, the highest values are in the last column, hence showing strong preference for the alters with same or higher values of PA. In group B, it is the opposite of group A, all egos preferred strictly smaller PA values (Table 6). 
Table 4: Group B Selection Effects: Estimates and standard errors

\begin{tabular}{ccc}
\hline Effect & Estimate & s.e. \\
\hline network rate period $1^{* *}$ & 4.5888 & 0.7446 \\
network rate period $2^{* *}$ & 3.7128 & 0.6488 \\
network rate period $3^{* *}$ & 9.2826 & 1.7696 \\
outdegree** & -3.1781 & 0.1379 \\
reciprocity** & 3.3751 & 0.2081 \\
transitive triplets** & 0.4125 & 0.0552 \\
int alter** & 0.4230 & 0.1426 \\
PA alter & -0.0691 & 0.1532 \\
\hline
\end{tabular}

Table 5: Group A ego - alter selection table for PA

\begin{tabular}{ccccc}
\hline$v_{i} / v_{j}$ & 1 & 2 & 3 & 4 \\
\hline 1 & -0.29 & -0.15 & -0.01 & 0.12 \\
2 & -0.16 & 0.58 & 0.72 & 0.86 \\
3 & -0.03 & 0.71 & 1.45 & 1.59 \\
4 & 0.09 & 0.84 & 1.58 & 2.33 \\
\hline
\end{tabular}

Table 6: Group B ego - alter selection table for PA

\begin{tabular}{ccccc}
\hline$v_{i} / v_{j}$ & 1 & 2 & 3 & 4 \\
\hline 1 & -0.18 & -0.24 & -0.31 & -0.37 \\
2 & 0.34 & -0.25 & -0.32 & -0.38 \\
3 & 0.86 & 0.26 & -0.33 & -0.39 \\
4 & 1.38 & 0.79 & 0.20 & -0.40 \\
\hline
\end{tabular}

We next examine the social influence processes for both groups, where we test for linear and quadratic shape effects, total and average similarity, indegree and outdegree and average alter effect for PA and NA. This is also where we look for any contagion effects and corresponding differences in the groups. 
We found that the average alter effect of PA in Group A was positive and significant, suggesting that actors adjust their PA to the higher values that their neighbours possess, through influence (rather than through creation of ties with similar individuals). This indicates contagion of PA between members of Group A. In the Group B, conversely, we found a significant effect for NA average alter, implying a contagion in NA ${ }^{10}$. This finding is highly significant, and we discuss it separately below.

Table 7: Group A Influence Effects: Estimates and standard errors

\begin{tabular}{ccc}
\hline Effect & Estimate. & s.e. \\
\hline rate PA period $1^{* *}$ & 3.2644 & 1.8913 \\
rate PA period $2^{* *}$ & 3.5390 & 1.3878 \\
rate PA period $3^{* *}$ & 3.5494 & 1.3137 \\
PA linear shape** & -0.3321 & 0.1333 \\
PA quadratic shape** & -0.3275 & 0.1817 \\
PA average alter** & 1.0379 & 0.5151 \\
PA effect from SWLS** & -0.8485 & 0.3078 \\
PA effect from gratitude** & 1.1409 & 0.4241 \\
PA effect from happiness & 0.8636 & 0.5003 \\
rate NA period 1 & 15.2069 & 20.2181 \\
rate NA period 2 & 1.4901 & 0.6995 \\
ate na (period 3 ) & 3.7385 & 2.9191 \\
NA linear shape & -1.5022 & 0.2772 \\
NA quadratic shape & 0.1087 & 0.2770 \\
NA average alter & 0.9990 & 0.7486 \\
\hline
\end{tabular}

The above provides a more complex picture of intra-group dynamics of PA and NA, which can be summarized in the following results.

R II.2 Contagion of positive affect is seen in group A.

R II.3 Contagion of negative affect is seen in group B.

\footnotetext{
${ }^{10}$ Results obtained by Neyman-Rao type score tests confirmed this result, with a p-value of 0.007 for PA average alter in group A and p-value of 0.025 for NA average alter in group B.
} 
Table 8: Group B Influence Effects: Estimates and standard errors

\begin{tabular}{ccc}
\hline Effect & Par.est. & s.e. \\
\hline rate PA period $1^{* *}$ & 2.9357 & 1.0676 \\
rate PA period 2** & 2.2314 & 0.6957 \\
rate PA period $3^{* *}$ & 1.7822 & 0.6513 \\
PA linear shape** & -0.3237 & 0.1139 \\
PA quadratic shape** & -0.2277 & 0.1149 \\
PA effect from SWLS** & 0.4027 & 0.2251 \\
rate NA period 1 & 3.6488 & 3.4166 \\
rate NA period 2 & 2.1090 & 0.9706 \\
rate NA period $3^{* *}$ & 17.3388 & 4.2537 \\
NA linear shape** & -2.2035 & 0.4536 \\
NA quadratic shape & 0.6475 & 0.4250 \\
NA average alter** & 1.3057 & 0.6536 \\
\hline
\end{tabular}

In terms of the exogenous covariates, SWLS had a negative influence on the PA dynamics in Group A. This implies that individuals with higher levels of SWLS had stronger tendency towards lower PA in group A, but no such effect could be detected in group B. This may seem a bit surprising, but a possible explanation may be found when we look at changes in PA, NA and the covariates in both groups and relate these to the pre-intervention levels (see Section 5.3.2). Happiness and Gratitude had small but significant positive effect on PA in Group A, but no such effect was found in B.

We also included a dummy variable as a covariate, that corresponded to being a part of the intervention (taking a value of 1 for participants chosen for the intervention and 0 for all the others). We included it in both the selection and influence tests of the model and found evidence of impact only for selection in Group B: a positive coefficient for alter-intervention, implying the tendency that intervention participants were selected with a higher likelihood than non-intervention participants, all other things being equal (see Table 4), but keeping in mind that participants were chosen based on their NA levels. The result obtained by NeymanRao type score test confirmed this with a p-value of 0.0042 for the intervention alter covariate. 
However, the results from the influence part of the model where we tested the alter's covariate average effect on PA and NA were not significant in any of two groups, and thus we were unable to prove that the agents of change were themselves directly influential.

\subsubsection{Intervention versus Baseline}

As in Study I, in the absence of an explicit control group, comparisons were made between the pre and post intervention measures of all variables. Hence we carried out the SIENA analysis on data from days 1 and 2 (baseline) and compared the results with the same analysis from days 3 and 4. Unlike Study I, no significant changes were detected between the two sets of data (apart from significant magnitude difference of variable transitivity triplets in selection effects).

Given that we detected contagion within both networks between days 1 to 4 (R II.2 and R II.3), we wanted to examine whether the contagion is related to the actual administration of the intervention or if it is an artefact of the data we generate. For this, we compared the results of the analysis carried out on day-pairs 1-2, 2-3 and 3-4, using the same set of variables in each. Neyman-Rao type generalized score tests on PA average alter for Group A and NA average alter for Group B (both, under the null hypothesis that the effect is zero) reveals an interesting pattern. The PA average alter effect (evidence for contagion) in group A and the NA average alter effect in group B are both significant between days 2 and 3 but not in other intervals. As a consequence, we are unable to rule out the possibility that PA and NA contagion are artefacts. Table 9 reports the corresponding p-values of the score tests for all pairwise analyses in both groups.

Table 9: Generalized score test p-values for testing existence of PA and NA contagion in consecutive day pairs.

\begin{tabular}{l|c|c}
\hline Day Pairs & $\begin{array}{c}\text { Group A p-values } \\
\text { PA average alter }\end{array}$ & $\begin{array}{c}\text { Group B p-values } \\
\text { NA average alter }\end{array}$ \\
\hline $1-2$ & 0.7863 & 0.2146 \\
$2-3$ & $0.0046^{* * *}$ & $0.0371^{* *}$ \\
$3-4$ & 0.5025 & 0.1341 \\
\hline
\end{tabular}


Following Study I, we used standard statistical comparisons to determine the direct effects of intervention on the various measures of well being. We applied the same non-parametric Wilcoxon Signed Rank test to assess changes in mean values of SWLS, Gratitude, Happiness, PA and NA between pre and post intervention time points for both groups $\mathrm{A}$ and $\mathrm{B}$. We compared these values for those who received the intervention (referred as Int), those who did not (referred as Non) as well as each group as a whole (referred as $A l l$ ). We expected the intervention to have a greater impact on Int, and more so for group A than in group B, due to the recipients in A having a lower level of NA overall.

Note that the issue of non-independence of observations is also valid here, as it was in Study 1. Given that the observations come from a longitudinal evolving network, the Wilcoxon test based p-values cannot be claimed as accurate indicators of statistical significance, but are provided as a guidance only. However, as Study 2 by nature is more complex that Study 1, we discuss this issue in greater detail below.

Table 10: Mean value of covariates in Group A - SLWS, Gratitude and Happiness.

\begin{tabular}{l|ll|ll|ll} 
& \multicolumn{2}{|c|}{ SWLS } & \multicolumn{2}{c|}{ Gratitude } & \multicolumn{2}{c}{ Happiness } \\
& pre & post & pre & post & pre & post \\
\hline All & 24.2 & 24.2 & 31.0 & $30.2^{* *}$ & 19.2 & 19.0 \\
Non & 23.9 & 21.6 & 30.9 & $27.4^{* * *}$ & 19.3 & 17.2 \\
Int & 25.1 & 26.1 & 31.5 & 31.1 & 19.1 & 19.6 \\
\hline
\end{tabular}

Table 11: Mean value of covariates in Group B - SLWS, Gratitude and Happiness.

\begin{tabular}{c|ll|ll|ll} 
& \multicolumn{2}{|c|}{ SWLS } & \multicolumn{2}{c|}{ Gratitude } & \multicolumn{2}{c}{ Happiness } \\
& pre & post & pre & post & pre & post \\
\hline All & 25.4 & 25.0 & 31.3 & $30.4^{* *}$ & 19.9 & 19.8 \\
Non & 25.5 & 24.8 & 31.8 & $29.9^{* *}$ & 20.0 & 19.0 \\
Int & 25.9 & $24.0^{* *}$ & 31.0 & 30.5 & 19.4 & 19.2 \\
\hline
\end{tabular}

Unlike Study 1, the effect of interventions in Study II are more complex in nature. As seen in Tables 10 and 11, there seems to be an overall downward trend in all the well-being measures over the entire week-long period. This is most likely due to exogenous factors which 
Table 12: Changes in PA, NA for Groups A and B.

\begin{tabular}{l|llll|llll} 
& \multicolumn{4}{|c|}{ Group A } & \multicolumn{4}{c}{ Group B } \\
& PA pre & PA post & NA pre & NA post & PA pre & PA post & NA pre & NA post \\
\hline All & 32.3 & 27.8 & 21.3 & 19.3 & 32.4 & 28.7 & 21.6 & $19.4^{* * *}$ \\
Non & 31.3 & $26.3^{* * *}$ & 21.9 & 18.7 & 31.8 & $28.1^{* * *}$ & 21.1 & $18.3^{* * *}$ \\
Int & 35.3 & 32.9 & 19.7 & $16.1^{* * *}$ & 34.7 & $31.2^{* *}$ & 23.3 & 24.0 \\
\hline
\end{tabular}

are beyond our knowledge and beyond the scope of control in this analysis. This confounds the straightforward comparison of PA and NA in the groups and sub-groups to a large extent.

Our focus was on the difference between pre (Day 0) and post (Day 5) study measures, but we examined the patterns within the 4 main days of the study as well. We could detect no significant differences in the mean PA and NA levels across these 4 days, on a day to day comparison. However, there were definite changes in the pre and post study measures, which are reported in Table 12. Given the proportion of the group members who were administered the intervention (approximately 25\% ) and the nature of the intervention itself, contagion alone was not "powerful" enough to reverse the overall trends in background PA and NA.

However, we are able to note two consequences of the intervention. First of all for Group A (in which we see contagion of PA), the intervention was successful in checking the strong significant downward trend in PA. As shown in Table 12, Int do not exhibit a significant change in PA, while Non exhibits a significantly fall (at $\mathrm{p}=0.01$ ) between Days 0 and 5 . Hence, our observation that the intervention worked well for those receiving it in Group A (in the sense of reducing the loss in PA relative to the 'background decline'), but the contagion was not strong enough to slow the decline in others. Secondly in Group B (in which we see contagion of NA), we see a slight increase in NA in Int $(24.0-23.3=0.7)$ between the start and end of the study. But this is in contrast with the NA in Non, which actually fell significantly (18.3 $21.1=-2.8$ ). This leads us to infer that, given the overall decline in all background measures, an increase in NA for Int indicates that the intervention aimed at high NA individuals in Group B, actually made matters worse for themselves (as seen in increase in NA) as well as for others in the network (R II.3).

These findings, on the face of it, support the hypothesis $\mathbf{H 4}$, that the outcome of selective psychological interventions within networks depends crucially on who is being targeted and 
their individual emotional state at the time of the intervention. It is not enough to consider just the physical position of these individuals within the network, as the intervention could result in undesirable consequences if the correct individuals are not targeted. However, they also depend to some extent on the use of the Wilcoxon Signed Rank test to compare across observations. These findings are highly exploratory in nature - and while they provide some evidence of validity of $\mathbf{H 4}$ - requires further large scale and more involved studies to be explored fully. They do however, provide an interesting direction where such future studies can be focussed on - that is, the impact of targeting selected individuals in a network, based not purely on their location, but also on their behaviour.

\subsection{Communicability}

In order to validate our assumptions of the best broadcasters being people with the lowest NA, we computed the communicability matrix post-study and calculated broadcast and receive indices from the 4 daily interaction matrices using duration of contacts as weights. The sorted broadcast indices of participants in group A and B are shown in Fig 2. We were able to correctly predict (i.e. identify them for the intervention based on their low NA on the first day of the study) the 1st, 2nd and 4th ranked individuals in terms of their broadcast index in Group A and avoid the top ranked people in Group B. We checked correlations between the broadcast communicability indices and all the other measures, and were unable to find any other predictor of high values. This validates our finding in Study I with regard to NA scores and broadcasting tendency.

\section{Discussion and Future Work}

The two studies presented here examined the effects of a gratitude based intervention within a social network, on the structure of the network itself and on the overall mood dynamics of members, as well as the inter-relationship between the two. The interventions were aimed at improving the overall levels of group level emotion (increase positive affect and decrease negative affect) Two types of interventions were examined - first, when the intervention was administered to all members within a social network, and the second, when administered to a selected few. Results from the studies indicate the following key outcomes. 


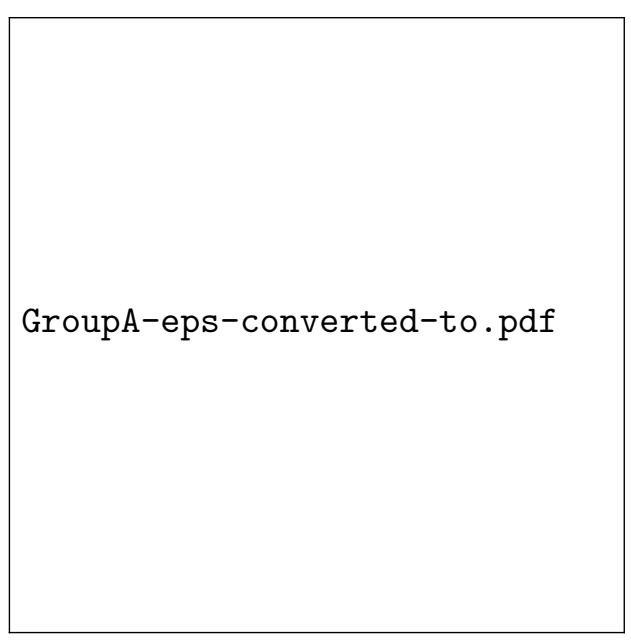

(a)

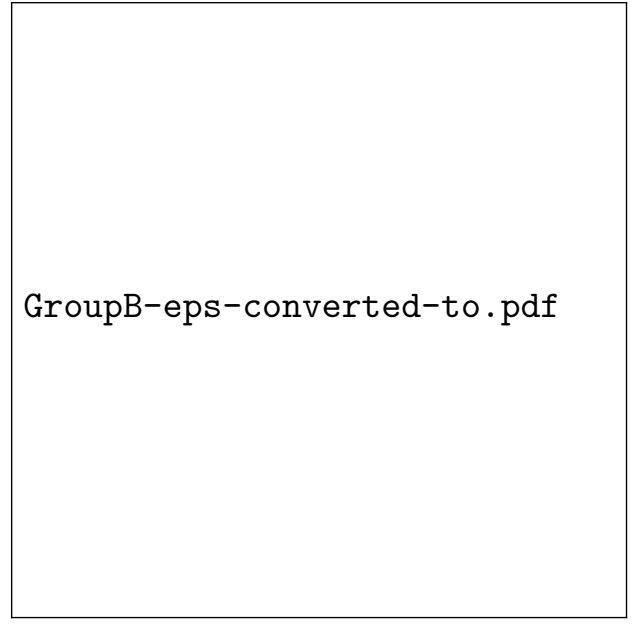

(b)

Figure 2: Panel (a): Sorted broadcast indices based on 4 days for individuals in Group A. Panel (b): Sorted broadcast indices based on 4 days for individuals in Group B. Black stars denote indices of participants who were part of the intervention.

1. The intervention was successful in achieving the desired group level effect when applied to the whole population. Homophily was also detected within the structure of the underlying social network, i.e. people initiated contact with others with similar levels of positive and negative affect. However, no additional evidence of contagion could be found in this case, where the intervention was applied uniformly to everyone.

2. The intervention was partially successful when applied to a quarter of a group, with evidence of contagion of positive affect in the group where the agents of change were chosen on the basis of low levels of initial negative affect. The contagion could not reverse the overall trends, but was successful in making the individuals who were administered the intervention more resilient to the trend (in the sense that the decrease in the mean was no longer significant).In addition we found that the contagion was not sustainable for longer time scales. We did not find evidence that it was triggered on by the intervention itself.

3. Adverse and unexpected (iatrogenic) effects were observed when intervention was administered selectively to the individuals with the highest negative affect - in the form of contagion of negative affect. There was an overall decrease of negative affect, but 
intervention group actually saw an increase in average negative affect (although not significant). Positive affect also decreased significantly, for both intervention and nonintervention group.

We are able to establish H1 (homophily) and H3 (desired improvement in group level mood) in a straightforward manner from Study 1, but we are unable to establish $\mathbf{H 2}$ (contagion of mood) from the data gathered in this study. However, Study 2 provides us with the evidence of contagion of both PA and NA, that was missing in Study 1, and also provides some indicative evidence of $\mathbf{H 4}$ (iatrogenic effects) at the same time. Thus, we can conclude that although intervention works when administered to a whole group, its effects spread only partially when administered only to a part of the group, and actually could possibly result in undesirable effects if not administered to the appropriate people (ones with low negative affect). Given these results, we have furthermore been able to establish contagion in mood related constructs within social networks - a phenomenon largely absent from previous behavioral studies of similar nature (Greetham et al. (2011)). It is only recently that we are able to observe both selection and influence effects in studies related to psychological constructs and social networks (Kalish et al. (2013)). Additionally, care needs to be taken when interpreting contagion results in Study 2. While we detected coincidence of intervention with contagion, we could not establish the direct causality between the interaction of those who received the intervention and the contagion effect observed.

It would be interesting to investigate why affect contagion was observed only in Study 2 and not in Study 1, whereas the reverse was observed for affect homophily. While the data did not reveal definite answers, we can conjecture that both effects were present in both studies in different proportions. This implies for instance, homophily was present in Study 2 within the subset receiving the intervention but the effect was too weak when the whole group was being considered, as the indirect impact on PA and NA was not strong enough. However, given the size of the samples and the nature of interactions, it is not possible to examine this separately. This is a conjecture which needs to be tested on larger and probably denser networks. In case of Study 1, it is more difficult to explain why contagion was absent while homophily was not, and requires further investigation as well. 
Note that both studies were run on students' existing social networks and network sizes were kept small in order to keep the study and the analysis manageable. We acknowledge that these social networks are not by any means complete from the point of view of the actors (participants), but we argue that they captured a large proportion of participants' social contacts during autumn term week-days given the number and the duration of contacts kept in diaries. While results cannot be automatically generalised to a wider population given that all our participants were second year students in two different UK universities, we hope that our studies might inspire further work on different demographics within existing "real" social networks such as schools, organisations, companies, retirement homes as well as on virtual ones such as Twitter and Facebook.

As we have seen in prior studies Golder and Macy (2011), PA, NA and other mood based constructs do exhibit short and medium term cyclical patterns, and hence our results are subject to these background changes as well. These may be further compounded by idiosyncratic exogenous effects as may be found among student groups in universities during term time. Moreover, the number of individuals subjected to the intervention was approximately one-quarter of the total group size. At this point, we have no way of judging a priori, whether the size of the chosen intervention group is optimum for the intervention being considered, given the nature of the underlying network. A deeper investigation on this topic alone is required for greater understanding and optimization of interventions. And finally, we chose a 4 day period for the studies, under the assumption that individuals receiving the intervention are able to transfer PA and NA within this time period, given their interaction level and the underlying social network structure. This is an assumption which requires further testing as well.

\subsection{Future work}

It is increasingly being recognized that interventions within social networks can potentially offer significant benefits in inducing sustainable behavior change Valente (2012). The most relevant examples lie in the health and well being area. However, additional important applications lie within organizational studies (improving work culture, motivation etc.), in education (in schools), public policy and health, in encouraging pro-environmental sustainable behavior, tackling crime and many others. This is an emerging field which is developing rapidly and we 
see the direction of our future work shaping in two complementary streams:

1. Designing small-scale controlled experiments in order to investigate optimal designs and contexts of networks based interventions in more depth (using disentanglement of influence/selection mechanisms to steer to desirable directions and impact of selecting subsets of individuals for intervention).

2. Validating our results on large-scale data-sets involving many participants and more dense networks.

We have already mentioned that further optimization is required as far as delivering interventions is concerned to a subset of nodes within a network. Related to that, further investigation is required on how to choose the agents of change. Our study found a relationship between NA and broadcasting, this needs to be validated further. Hence more work is necessary to understand this relationship more thoroughly. Further work could be done in relation to larger/finer time-scales and the length of the time-window in which these observations stay valid etc. Moreover, we have only chosen the 'individual' based intervention strategy - whereas at least three more strategies exist (and many more if they are combined) which can be used to deliver the intervention (see Valente (2012) for more details). We are also aware of the design limitation of the studies presented here - in that, they have been carried out in student based environments exclusively and they rely on self-reporting, so due caution is called for when interpreting the results (as in every other study with self-reported behaviour).

\section{Acknowledgments}

This work was fully funded by Unilever. We would like to thank to Dr. Alex Linley for helping with the design and collecting the data for the first study and to Emily Hancock who collected the data for the second study. We would also like to extend our thanks to the anonymous referee who provided us with excellent comments during the peer review stage. 


\section{A Details on SIENA}

Analysis of empirical longitudinal networks involve studying the evolution of the structure of the network, and its interaction with individual characteristics and associated behaviour on part of the "actors" or members of the network. The so called actor-oriented models are designed to identify the effect of these characteristics on the evolutionary nature of the network, under the assumption that the formation of the network is a stochastic process, where network autocorrelation arises out of the inter-play between behavioural dimensions and structural dimensions, in the context of each actor making an individual decision regarding a tie or level of a certain behaviour at any given time Steglich et al. (2010). SIENA is one such analytical framework based on stochastic actor-oriented models. Estimation in SIENA involves identifying parameter estimates of factors affecting network structure as well as underlying actor behaviour (using a simultaneous equation framework) and then using agent based simulations for inference.

Specification of a model constructed using SIENA usually involves objective functions (network structural properties thought to cause the evolution of the network, which can be specified in the software choosing existing structural or attribute based factors or creating a new combinations of such factors) and rate functions - the estimated number of changes each individual can make between observed time points. The input consists of matrices of network tie data (each matrix representing a wave) for all nodes, attribute vectors that may be constant (demographics or measures that didn't change during the study) and dependent variables such as a measured behaviour that we are interested in. SIENA is a package within the $\mathrm{R}$ statistical framework. Once run on the given data, SIENA assesses the impact of the various input variables - structural (e.g. reciprocity, density) and attributes (e.g. age, behaviour). Given that actor based models assume that at any given moment, not more than one tie variable or one behaviour variable can change, the actual resultant dynamics (order of network creation and behaviour evolution) is too complex for direct classical estimation procedures. Hence SIENA uses an agent based simulation approach to determine parameter estimates as those values under which the simulated and observed data resemble each other most closely (convergence. The model convergence is verified using standard t-tests. Furthermore, the estimation assessment is supported through generalized Neyman-Rao score tests as well. 
Here we list the main effects were included in the objective function in our analysis (as defined in Ripley and Snijders (2011)). We used RSiena4 (version 1.1.232) for all analysis. The objective functions (network and behaviour) for actor $i$ included following effects:

\section{A.0.1 Structural effects}

- out-degree effect, defined by the out-degree $s_{i 1}^{n e t}(x)=x_{i+}=\sum_{j} x_{i j}$, where $x_{i j}=1$ indicates presence of a tie from $i$ to $j$ while $x_{i j}=0$ indicates absence of this tie;

- reciprocity effect, defined by the number of reciprocated ties, $s_{i 2}^{\text {net }}(x)=\sum_{j} x_{i j} x_{j i}$;

- transitive triplets effect, defined by the number of transitive patterns in $i$ 's relations (ordered pairs of actors $(j, h)$ to both of whom $i$ is tied, while also $j$ is tied to $h$ ), for directed networks $s_{i 3}^{n e t}(x)=\sum_{j, h} x_{i j} x_{i h} x_{j h}$.

\section{A.0.2 Covariate effects}

- covariate-alter effect, defined by the sum of the covariate over all actors to whom $i$ has a tie, $s_{i 4}^{n e t}(x)=\sum_{j} x_{i j} v_{j}$, where $v_{j}$ is an actor-dependent covariate or dependent behaviour variable;

- covariate-ego effect, defined by $i$ 's out-degree weighted by his covariate value, $s_{i 5}^{\text {net }}(x)=$ $v_{i} x_{i+}$

- covariate-related similarity, defined by the sum of centered similarity scores $\operatorname{sim}_{i j}^{v}$ between $i$ and the other actors $j$ to whom he is tied, $s_{i 6}^{n e t}(x)=\sum_{j} x_{i j}\left(\operatorname{sim}_{i j}^{v}-\operatorname{sim}^{v}\right)$.

\section{A.0.3 Behavioural effects}

- behavioral shape effect (linear), $s_{i 1}^{b e h}(x, z)=z_{i}$, where $z_{i}$ denotes the value of the dependent behavior variable of actor $i$;

- quadratic shape effect, or effect of the behavior upon itself, where the attractiveness of further steps up the behavior 'ladder' depends on where the actor is on the ladder: $s_{i 2}^{b e h}(x, z)=z_{i}^{2}$; 
- average alter effect, defined by the product of $i$ 's behaviour multiplied by the average behaviour of his alters $\operatorname{sim}_{i j}^{z}$ between $i$ and the other actors $j$ to whom he is tied, $s_{i 15}^{b e h} z_{i}\left(\sum_{j} x_{i j}, z_{j}\right) /\left(\sum_{i} x_{i j}\right)$.

- average similarity effect is defined by the average of centered similarity scores between $i$ and the other actors $j$ to whom he is tied, $s_{i 3}^{b e h}(x)=x_{i+}^{-1} \sum_{j} x_{i j}\left(\operatorname{sim}_{i j}^{z}-s_{i m^{z}}\right)$.

- total similarity effect is defined by the sum of centered similarity scores between $i$ and the other actors $j$ to whom he is tied, $s_{i 3}^{b e h}(x)=\sum_{j} x_{i j}\left(s i m_{i j}^{z}-s i \hat{m}^{z}\right)$.

\section{A.0.4 Covariate effects on behaviour}

For each actor-dependent covariate or dependent behavior variable $v_{j}$ (which are centered)

- main covariate effect, $s_{i 4}^{b e h}(x, z)=z_{i} v_{i}$.

\section{B Data - Study I}

All 89 students were undergraduate students from the same discipline and cohort in a residential campus based University. We used age, gender and ethnicity as constant covariates. Average age was 20.05, a minimum age was 19 and a maximum was 23 . There were 76 female and 13 male participants, coming from 6 different ethnical backgrounds. Around $72 \%$ participants declared White, around 16\% Indian, 4.5\% Black African, 2.3\% each Black Caribbean and Chinese, and three other ethnicities had only one representative each so we marked them as Other.

All measurements were carried out during term time, and hence we expected that most of the social interactions of participants would be restricted to each other, both in and out of class. A summary of interactions is presented below in Table 13. We recognise that the participants would most likely have interacted outside this network, but we expect that these were minimal, given the timing of the study. In any case, it would not be feasible to track all social interactions of such a group of individuals. As the measures of times spent interacting with each other were self reported, we validated reports by matching them against each other. For instance, if participant 1 reports $\mathrm{X}$ amount of time spent interacting with participant 2 on a given day, we would expect to see the same report from participant 2 about interaction 
with 1 . The validation did throw up some minor discrepancies, but these could be ignored given their magnitudes.

Table 13: Study I: Total time spent individually by participants in social interactions within the group per day (self reported).

\begin{tabular}{l|llll} 
& Day 1 & Day 2 & Day 3 & Day 4 \\
\hline Min & 0 & 0 & 0 & 0 \\
Max & 1036 & 1110 & 1397 & 2070 \\
Mean & 163 & 131.6 & 159 & 231.9 \\
Std. Dev. & 248.7 & 213.0 & 243.3 & 375.8 \\
\hline
\end{tabular}

\section{Data - Study II}

As in Study I, each group was drawn from the same year and degree course. We used age, gender and ethnicity as constant covariates. In group A, average age was 20.20, standard deviation based on a sample 2.92, a minimum age was 19 and a maximum was 40 . There were 47 female and 15 male participants, coming from several different ethnic backgrounds. Around $63 \%$ participants declared as White British, around 12\% White, 3\% Chinese, $3 \%$ Indian and three other ethnicities had only one representative each. In group B, average age was 20.20, standard deviation based on a sample 2.60, a minimum age was 19 and a maximum was 32 . There were 60 female and 5 male participants. Around $70 \%$ participants declared as White British, around 11\% British, around 10\% white and several other ethnicities that had only one representative. A summary of interactions is presented below in Table 14. As in Study I, all measurements were carried out during term time, and hence we expected that most of the social interactions of participants would be restricted to each other, both in and out of class. Although there is a difference in a time spent in contacts, especially in the first two days between groups $\mathrm{A}$ and $\mathrm{B}$, the total number of links in daily interaction matrices used for SIENA was for consecutive days 182, 177, 123 and 142 for A and 160, 104, 119, 170 for B, and thus should not have significantly affect results, as Jaccard coefficients in the groups A and B were respectively $0.496,0.415,0.387$ and $0.392,0.429,0.363$, thus still in the limit of a allowed level of a network change for SIENA show. 
Table 14: Study II: Total time spent individually by participants in social interactions within the group per day (self reported).

\begin{tabular}{l|llll|llll} 
& \multicolumn{5}{|c}{ Group A } & \multicolumn{5}{c}{ Group B } \\
& Day 1 & Day 2 & Day 3 & Day 4 & Day 1 & Day 2 & Day 3 & Day 4 \\
\hline Min & 0 & 0 & 0 & 0 & 0 & 0 & 0 & 0 \\
Max & 1225 & 1360 & 1390 & 1020 & 607 & 670 & 2414 & 1210 \\
Mean & 293 & 285.1 & 207.1 & 267.7 & 147.2 & 136.2 & 295.9 & 292.9 \\
Std. Dev. & 294.4 & 284.7 & 278.6 & 288.6 & 148.1 & 168.9 & 504.7 & 282.7 \\
\hline
\end{tabular}

\section{The PANAS scale}

Here we provide details of the 20 item PANAS scale (provided before and after the study) and the daily version, which consisted of 10 items.

\section{D.1 20 item PANAS}

This scale consists of a number of words that describe different feelings and emotions. Participants were asked to read each item and indicate on a scale of 1 to 5 , to what extent they experienced each in the past one week. The scales represented the following: 1 - very slightly, 2 - a little, 3 - moderately, 4 - quite a bit and 5 - extremely. The items were:

1. Interested 2. Irritable 3. Distressed 4. Alert 5. Excited 6. Ashamed 7. Upset 8. Inspired 9. Strong 10. Nervous 11. Guilty 12. Determined 13. Scared 14. Attentive 15.Hostile 16. Jittery 17. Enthusiastic 18. Active 19. Proud 20. Afraid

\section{D.2 10 item PANAS}

The 10 item scale consisted of the following:

1. Interested 2. Irritable 3. Distressed 4. Excited 5. Inspired 6. Nervous 7. Hostile 8. Attentive 9. Jittery 10. Enthusiastic

The participants were once again asked to rate each item on a scale of 1 to 5 . 


\section{References}

Abraham, C. and S. Michie (2008, May). A taxonomy of behavior change techniques used in interventions. Health Psychology 27(3), 379-387.

Barsade, S. G. (2002). The ripple effect: Emotional contagion and its influence on group behavior. Administrative Science Quarterly 47(4), 644-675.

Borgatti, S. and M. Everett (2006). A graph-theoretic perspective on centrality. Social Networks 28(4), 466-484.

Cross, R. and A. Parker (2004). The Hidden Power of Social Networks: Understanding How Work Really Gets Done in Organizations. Harvard Business School Press.

DeNeve, K. M. and H. Cooper (1998). The happy personality: a meta-analysis of 137 personality traits and subjective well-being. Psychological Bulletin 124, 197-229.

Diener, E. and R. Emmons (1984). The independence of positive and negative affect. J Pers Soc Psychol. 47(5), 1105-17.

Diener, E., R. A. Emmons, R. J. Larsen, and S. Griffin (1985). The satisfaction with life scale. Journal of Personality Assessment 49, 71-75.

Duerr, H., M. Schwehm, C. Leary, S. d. Vlas, and M. Eichner (2007). The impact of contact structure on infectious disease control: influenza and antiviral agents. Epidemiology and Infection 135(7), 1124-1132.

Ellwardt, L., C. Steglich, and R. Wittek (2012). The co-evolution of gossip and friendship in workplace social networks. Social Networks 34(4), 623-633.

Emmons, R. and M. McCullough (2003a). Counting blessings versus burdens: An experimental investigation of gratitude and subjective well-being in daily life. Journal of Personality and Social Psychology 84, 377-389.

Emmons, R. and M. McCullough (2003b). The gratitude questionnaire-six item form (gq-6). "http://www.psy.miami.edu/faculty/mmccullough/gratitude/GQ-6.pdf".

Estrada, E. and N. Hatano (2008). Communicability in complex networks. Physical Review E 77 . 
Fordyce, M. (2005). A review of research on the happiness measures: A sixty second index of happiness and mental health. In A. Michalos (Ed.), Citation Classics from Social Indicators Research, Volume 26 of Social Indicators Research Series, pp. 373-399. Springer Netherlands. Golder, S. and M. W. Macy (2011). Diurnal and seasonal mood tracks work sleep and daylength across diverse cultures. Science 333(6051), 1878-1881.

Gray, E. and D. Watson (2007, March). Assessing positive and negative affect via self-report, pp. 171-183. New York: Oxford University Press.

Greetham, D., A. Linley, R. Hurling, and G. Osborne (2011). Social networks and positive and negative affect. Procedia-Social and Behavioural Sciences, Dynamics of Social Network Analysis, 4-13.

Grindrod, P., D. J. Higham, M. C. Parsons, and E. Estrada (2011). Communicability across evolving networks. Physical Review E, 83.

Huisman, C. (2013). Does it matter what friends think, say or do? the role of friends' smoking attitudes and behavior for dutch adolescents' smoking behavior. Substance Use and Misuse.

Kalish, Y., G. Luria, and M. Westman (2013). Till stress do us part: Linking communication networks, stress and voluntary exit in extreme contexts. Mimeo: http://sonic.northwestern.edu/wp-content/uploads/2011/08/TILL-STRESS-DO-US-PARTNorthwestern.pdf.

Katz, L. (1953). A new index derived from sociometric data analysis. Psychometrika 18, $39-43$.

Kramer, A. D. I., J. E. Guillory, and J. T. Hancock (2014). Experimental evidence of massivescale emotional contagion through social networks. Proceedings of the National Academy of Sciences 111(24), 8788-8790.

La Guardia, J. G., C. E. Ryan, Richard M.and Couchman, and E. L. Deci (2000). Withinperson variation in security of attachment: A self-determination theory perspective on attachment, need fulfillment, and well-being. Journal of Personality and Social Psychology 79, $367-384$.

McPherson, J., L. Smith-Lovin, and J. Cook (2001). Birds of a feather: homophily in social 
networks. Annual Review of Sociology 27, 415Ü444.

Michie, S. (2008). Designing and implementing behaviour change interventions to improve population health. Journal of Health Services Research and Policy 13 (suppl 3), 64-69.

Niedenthal, P. M. and M. Brauer (2012). Social Functionality of Human Emotion. Annual Review of Psychology 63, 259-285.

Norman, G., M. Zabinski, M. Adams, D. Rosenberg, A. Yaroch, and A. Atienza (2007). A review of ehealth interventions for physical activity and dietary behavior change. American Journal of Preventive Medicine 33(4), 336Ü345.

Opsahl, T., F. Agneessens, and J. Skvoretz (2010). Node centrality in weighted networks: Generalizing degree and shortest paths. Social Networks 32(3), 245.

Quercia, D., L. Capra, and J. Crowcroft (2012). The social world of twitter: Topics, geography, and emotions. Dublin. The 6th International AAAI Conference on weblogs and social media. Ripley, R. and T. Snijders (2011). Manual for siena version 4.0. Technical report, Oxford,. Russell, D.and Peplau, L. and C. Cutrona (1980). The revised ucla loneliness scale: Concurrent and discriminant validity evidence. Journal of Personality and Social Psychology 39, 472-480. Schaefer, D. R., J. Adams, and S. Haas (2013). Social networks and smoking - exploring the effects of peer influence and smoker popularity through simulations. Health Education and Behavior $40(1 \mathrm{~S}), 24 \mathrm{~S}-32 \mathrm{~S}$.

Schaefer, D. R., J. M. Light, R. A. Fabes, L. D. Hanish, and C. L. Martin (2010). Fundamental principles of network formation among preschool children. Social Networks 32, 61-71.

Snijders, T., C. Steglich, and G. V. de Bunt (2010). Introduction to actor-based models for network dynamics. Social Networks 32, 44-60.

Snijders, T. A. (2005). Models for Longitudinal Network Data, Chapter 11, pp. 215-247. Cambridge University Press.

Steglich, C., T. A. B. Snijders, and M. Pearson (2010). Dynamic networks and behavior: Separating selection from influence. Sociological Methodology 40(1), 329-393.

Tellegen, A. (1985). Structures of mood and personality and their relevance to assessing anx- 
iety, with an emphasis on self-report, pp. 681Ü-706. Hilsdale.

Totterdell, P., T. Wall, D. Holmand, H. Diamond, and O. Epitropaki (2004). Affect networks: A structural analysis of the relationship between work ties and job-related affect. Journal of Applied Psychology 89, 854-867.

Valente, T. (2012). Network interventions. Science 337(6090), 49-53.

Valente, T. and P. Pumpuang (2007). Identifying opinion leaders to promote behavior change. Health Educ Behav 34(6), 881-896.

Wood, A., J. Froh, and A. Geraghty (2010). Gratitude and well-being: A review and theoretical integration. Clinical psychology review 30, 890-905. 